\title{
Comportement de la phase argileuse lors de la dessiccation dans des Ferralsols microagrégés du Brésil : rôle de la microstructure et de la matière organique
}

Luiz Carlos Balbino, a,b, Ary Bruand, c, Michel Brossard d, Maria de Fatima Guimarães, e

a Unité de science du Sol, Inra Orléans, BP 20619, 45166 Olivet Cedex, France

b Embrapa Arroz e Feijão, BP 179, 75375-000 Santo Antônio de Goiás, GO, Brésil

c Institut des sciences de la Terre d'Orléans, université d'Orléans, Géosciences, BP

6759, 45067 Orléans Cedex 2, France

d IRD/Embrapa Cerrados, BP 7091, 71619-970 Brasilia, DF, Brésil

e Universidade Estadual de Londrina, Departamento de Agronomia, BP 6001, 86051-

990 Londrina, PR, Brésil

\begin{abstract}
Behaviour of the clay fraction during desiccation in microaggregated Ferralsols from Brazil: role of microstructure and organic matter.

The physical properties of the microaggregates of Ferralsols are still under discussion. Two sites were selected for study: in each site, one soil under a native savannah (Cerrado) and another one under pasture were studied. Samples were analysed in the laboratory by using scanning electron microscopy and mercury porosimetry and by measuring the water content at -0.1 and $-1.5 \mathrm{MPa}$ potential. Results showed the presence of microaggregates 50 to $300 \mu \mathrm{m}$ in size and bigger ones 500 to $1000 \mu \mathrm{m}$ in size. The latter were often packed in dense volumes 2 to $20 \mathrm{~mm}$ in size, which were numerous in the top soil under pasture. These dense volumes are casts and would result from an imbalance in the faunal population as a consequence of deforestation. Results showed also a shrinkage of the clayey phase for drying and this shrinkage increased with the carbon content of the soil. These results showed that microaggregates are not rigid fabrics and the significant role of organic matter in physical properties of the soils studied.
\end{abstract}

microaggregate / shrinkage / clay / cerrado / organic carbon / termite / cast / Brazil

Résumé - Les propriétés physiques des microagrégats des Ferralsols sont encore largement discutées. Deux sites ont été sélectionnés et, pour chaque site, un sol sous une savane arborée (Cerrado) et un autre sous un pâturage ont été étudiés. Des échantillons ont été analysés en microscopie électronique à balayage et en porosimétrie au mercure ; on a mesuré la teneur en eau aux potentiels de $-0,1$ et $-1,5 \mathrm{MPa}$. Les résultats établissent la présence de microagrégats de 50 à $300 \mu \mathrm{m}$ et d'autres de 500 à $1000 \mu \mathrm{m}$, ces derniers étant fréquemment assemblés en volumes compacts de 2 à 20 
$\mathrm{mm}$. Ces volumes denses sont des turricules et leur proportion élevée sous pâturage résulterait d'un déséquilibre dans la faune du sol, à la suite du défrichement. Les résultats indiquent aussi que les microagrégats ne sont pas des édifices rigides hors de la dessiccation et mettent en évidence le rôle significatif de la matière organique dans ce comportement.

\title{
microagrégat I retrait I argile I cerrado I carbone organique I termite I turricule I Brésil
}

\begin{abstract}
Abridged version
Ferralsols that cover large areas under the Tropics have a clay fraction which is mainly low activity clay consisting in kaolinite [10]. Ferralsols show little or no distinct horizonation and their macro-structure is weak to moderate. The ferralic horizon has typically a strong microstructure that consists in near spherical microaggregates ranging 100-200 $\mu \mathrm{m}$ in size. Microaggregates are considered as stable to highly stable by many authors. Pinheiro-Dick and Schwertmann [17] showed that clay dispersion was completed only after oxi-hydroxide removal. Thus, the amount of water-dispersible clay should be often very low in Ferralsols. However other studies showed that the clay fraction could be easily dispersed [12]. Hartmann [9] showed that dispersion of the microaggregates was recorded because of the effect of $\mathrm{K}+$ as dispersing agent. A recent study confirmed that the presence of microaggregates would be a characteristic common to most of Ferralsols [11], but we have little information about the physical properties of the microaggregates. The aim of this Note is to discuss the behaviour of the microaggregate porosity by comparing the porosity of wet microaggregates at -0.1 and $-1.5 \mathrm{MPa}$ water potential with its value after drying. Two sites located in the Brazilian savannah (Cerrado) were selected for study. The climate in the Cerrado region is characterized by distinct wet and dry seasons. One site is located in the state of Mato Grosso do Sul (Brasilândia) and the other site in the state of Goiás (Planaltina). The soil developed on Cretaceous sandstone (Baurú group) at the site of Brasilândia and on Meso-Neoproterozoic metasediments (Paranoá group) at the site of Planaltina. The soils are Latossolos vermelho [6] or Ferralsols [10]. The soil under two land uses was studied at each site : (i) under a native savannah (Cerrado), which was a forest, and (ii) under a low productivity pasture of Brachiaria decumbens at Brasilândia and of Brachiaria brizantha cv. Marandú at Planaltina.
\end{abstract}

The bulk density was measured at several depths. Because of the very low cohesion of the soil, small undisturbed samples were obtained in the laboratory using copper cylinders. They were used to investigate the pore size distribution and water retained at -0.1 and $-1.5 \mathrm{MPa}$ potential. The rest of the samples was air-dried and sieved at $2 \mathrm{~mm}$ for physico-chemical analyses. We measured the particle size distribution by using $\mathrm{NaOH}$ and hexametaphosphate buffered with $\mathrm{NaCO} 3$ [3]. Organic carbon content was measured by oxidation and the cation exchange capacity (CEC) using the cobalthexamine trichloride method [5]. The Fe and Al belonging to oxides and hydroxides was determined [13]. We measured the specific water content ( $W$ in $g$ of water per $g$ of ovendried soil) at -0.1 and $-1.5 \mathrm{MPa}$ water potential. Six replications were preformed for every horizon studied. The pore size distribution of the sample was determined in 
mercury porosimetry in triplicates. The surface tension of mercury and its contact angle with the soil material were taken as $0.484 \mathrm{~N} \cdot \mathrm{m}-1$ and $130 \circ$, respectively. The microstucture was studied also using the backscattered electron emission in scanning electron microscopy. Results showed that the clay content slightly increased with depth at Brasilândia site and remains approximately constant at Planaltina site (table). The cation exchange capacity decreased with depth from 3.3 to $1.8 \mathrm{cmolc} \cdot \mathrm{kg}-1$ at Brasilândia site and from 9.3 to $2.5 \mathrm{cmolc} \cdot \mathrm{kg}-1$ at Planaltina. The organic carbon decreased with depth but it was higher at every depth in the two soils of Planaltina than in those of Brasilândia (table). The free iron and aluminium content was much higher in Planaltina than in Brasilândia. Scanning electron microscopy showed the presence of two types of microaggregates: subcircular microaggregates 50 to $300 \mu \mathrm{m}$ in size which were juxtaposed and bigger microaggregates 500 to $1000 \mu \mathrm{m}$ in size which were often closely packed in aggregates 2 to $20 \mathrm{~mm}$ in size (figure 1). The proportion of microaggregates 50 to $300 \mu \mathrm{m}$ in size increased with depth in the soils studied. Observations showed also that lots of microaggregates 500 to $1000 \mu \mathrm{m}$ in size in close packing were present between 3 and $40 \mathrm{~cm}$ depth in the soil under pasture at Planaltina site. In Brasilândia, a higher proportion of these microaggregates in close packing was also noticed between 6 and $12 \mathrm{~cm}$ depth under pasture but appeared less developed. In Brasilândia, the water retained at -0.1 and $-1.5 \mathrm{MPa}$ water potential ranged from 0.087 and $0.167 \mathrm{~cm} 3 \cdot \mathrm{g}-1$ and between 0.066 and $0.129 \mathrm{~cm} 3 \cdot \mathrm{g}-1$, respectively (table). In Planaltina, the water retained at -0.1 and -1.5 MPawater potential ranged from 0.248 and $0.304 \mathrm{~cm} 3 \cdot \mathrm{g}-1$ and between 0.223 and $0.246 \mathrm{~cm} 3 \cdot \mathrm{g}-1$, respectively (table). Mercury intrusion curves showed the presence of two classes corresponding to pores with $0.006<D e<0.2 \mu \mathrm{m}$ and $0.2<D e<360 \mu \mathrm{m}$, respectively. We will discuss here the volume of pores with $\mathrm{De}_{-} 3 \mu \mathrm{m}$ (table). The microaggregates 50 to $300 \mu \mathrm{m}$ in size correspond to the microaggregates that were described for the ferralic horizon [10] and would result from termite activity $[7,15]$. The bigger microaggregates that observed isolated or packed in dense volumes are casts produced by a small geophageous earthworm. The high proportion of these dense volumes between 3 and $40 \mathrm{~cm}$ of depth under pasture at Planaltina site would correspond to physical transformation of the soil. It would be related to an imbalance in the faunal population as a consequence of deforestation. Such an evolution has similarities with the one already recorded after deforestation in Amazonia [4]. Under pasture at Brasilândia site, the higher proportion of dense volume that was recorded between 6 and $12 \mathrm{~cm}$ depth would correspond to similar physical modification. The amount of water retained at -0.1 and $-1.5 \mathrm{MPa}$, potential is closely related to the clay content $(r 2=0.95$ and 0.99 , respectively), which is consistent with the location of water at $-1.5 \mathrm{MPa}$ within the pores resulting from the packing of clay particles. At -0.1 and $-1.5 \mathrm{MPa}$, one can consider that the water is retained by pores with equivalent pore diameter $\leq 3$ and $\leq 0.2 \mu \mathrm{m}$, respectively [14]. As earlier done [2], we compared the volume of water retained at -0.1 and $-1.5 \mathrm{MPa}$ and the volume of these pores after drying. Whatever the soil studied, the volume of water retained at -0.1 and $-1.5 \mathrm{MPa}$ is higher than the volume of pores with $D e \leq 3$ and 0.2 $\mu \mathrm{m}$, respectively. The pore volume in the dried soil was 3.7 to $31.7 \%$ less than the volume of water at -0.1 and 6.6 to $29.9 \%$ less than the volume of water at $-1.5 \mathrm{MPa}$, thus indicating a shrinkage of the clayey phase for drying. The observations in scanning electron microscopy showed that the proportion of the different types of microaggregates 
and their packing varied within the soils studied (figure 1). Results showed the lack of difference according to the characteristics of the microstructure, thus indicating that the shrinkage measured is a property of the clayey phase whatever its organisation in the different types of microaggregates. When we analyse the shrinkage intensity between $-0.1 \mathrm{MPa}$ and the dried state and between $-1.5 \mathrm{MPa}$ and the dried state, there is no relationship with the depth and the clay content but a close relationship with the carbon content (figure 2a). Indeed the shrinkage increased with the carbon content. As indicated by the $\mathrm{C} / \mathrm{N}$ ratio (table), the organic matter can be considered as mainly composed by highly transformed organic matter and closely associated with the clayey phase [8]. Thus organic matter molecules would shrink during drying and lead to a closer packing of the clay particles thus inducing a shrinkage and a decrease in the pore volume. The results presented in this paper showed that microaggregates in Ferralsols are not rigid fabric and shrink for drying. This shrinkage of the microaggregates might occur in the whole range of water potential should be quantified for a better understanding of the structure dynamics in Ferralsols. Finally, the close relationship that was recorded between the shrinkage intensity of the clayey phase and the carbon content of the soil showed that the organic matter play a key role in the physical properties of the soil studied.

\section{Introduction}

Les Ferralsols résultent d'une longue et intense altération et représentent environ 750 millions d'hectares dans la zone intertropicale [10]. La fraction argile est composée essentiellement de kaolinite et, secondairement, d'oxy-hydroxydes de fer. Les Ferralsols présentent une faible différenciation en horizons et la macrostructure est peu développée, voire absente. Leur microstructure est, en revanche, très nette et représentée par des microagrégats de forme sphérique et de 100 à $200 \mu \mathrm{m}$ de diamètre. Les microagrégats sont considérés par certains auteurs comme étant très stables, en raison des forces électrostatiques développées entre les oxy-hydroxydes de fer chargés positivement et les particules de kaolinite possédant des charges négatives [16]. La dispersion des microagrégats ne serait alors obtenue qu'après avoir éliminé les oxy-hydroxydes de fer, comme l'ont rapporté Pinheiro-Dick et Schwertmann [17] pour des Ferralsols du Brésil et du Cameroun. Pour d'autres, en revanche, les microagrégats peuvent être dispersés après saturation des sites d'échange avec $\mathrm{Na}+$, révélant ainsi que l'argile peut être dispersée en utilisant $\mathrm{Na}+$ comme seul dispersant [12]. Hartmann [9], étudiant des Ferralsols en Côte-d'Ivoire, a montré que l'apport d'ions K+ suffit pour disperser l'argile des microagrégats et conduire à la formation d'une matrice argileuse massive. Une étude des Ferralsols appartenant à la collection de sols du Centre international d'information et de référence sur les sols (ISRIC, Wageningen) a permis de comparer des Ferralsols issus d'Amérique du Nord et du Sud, d'Afrique et d'Asie [11]. Cette étude conclut que la présence de microagrégats est bien un caractère commun à l'ensemble des Ferralsols étudiés et que ceux-ci résulteraient des effets combinés de l'activité biologique et d'une composition minéralogique particulière. En revanche, aucun élément n'est apporté quant aux propriétés physiques des microagrégats, hormis leur stabilité élevée lors d'une agitation dans l'eau. L'objectif de cette note est d'apporter des données nouvelles sur les propriétés physiques des microagrégats, en particulier sur leurs propriétés de retrait lorsque l'état hydrique du sol varie. 


\section{Les sols étudiés}

Les sols étudiés sont localisés dans la région du Cerrado. II s'agit de Ferralsols [11], correspondant à des Latossolo vermelho [6]. Le climat se caractérise par une pluviosité annuelle de 1200 à $1600 \mathrm{~mm}$, avec une saison humide et une saison sèche très distinctes, 80 à $90 \%$ de la pluviosité annuelle étant enregistré entre les mois d'octobre et d'avril. Dans un premier site localisé dans l'État du Mato Grosso do Sul (Brasilândia, 21'22' S, 52.11' W), deux sols ont été sélectionnés, l'un dans une zone de savane de type Cerrado, et l'autre dans une zone de pâturage de Brachiaria decumbens, semée après déforestation en 1974. Dans un second site localisé dans l'État du Goiás, deux autres sols ont été sélectionnés (Planaltina, 15॰14' S, 47॰42' W), l'un dans une zone de savane de type Cerrado, l'autre dans un pâturage de Brachiaria brizantha c.v. Marundú, semée en 1990 après défrichement. Les sols du site de Brasilândia sont développés sur des grès d'âge Crétacé (groupe Baurú) et ceux du site de Planaltina sur des sédiments métamorphisés d'âge Méso-Néoprotérozoïque (groupe Paranoá). Les sols présentent les caractères morphologiques des Ferralsols : des horizons difficilement identifiables, une macrostructure très faiblement développée à absente, et une microstructure très nette, sous la forme de microagrégats sub-sphériques de 200 à $500 \mu \mathrm{m}$ de diamètre. Cette microstructure est néanmoins moins développée dans les sols sous pâturage, en particulier entre 6 et $12 \mathrm{~cm}$ de profondeur à Brasilândia et entre 3 et $40 \mathrm{~cm}$ de profondeur à Planaltina.

\section{3. Échantillonnage et méthodes d'étude}

Des blocs de dimensions pluri-décimétriques ont été prélevés sur le terrain. En raison de la très faible cohésion du sol, des échantillons de petite taille ont ensuite été prélevés à différentes profondeurs dans les blocs à l'aide de cylindres en cuivre de $13 \mathrm{~cm} 3$. Pour chaque profondeur échantillonnée, le reste du bloc a ensuite été séché à l'air et tamisé à $2 \mathrm{~mm}$ pour les analyses physico-chimiques. L'analyse granulométrique a été réalisée après destruction de la matière organique avec $\mathrm{H} 2 \mathrm{O} 2$ pour les échantillons prélevés entre 0 et $20 \mathrm{~cm}$ de profondeur et dispersion avec $\mathrm{NaOH}$ et de l'hexamétaphosphate de sodium pour l'ensemble des échantillons [3]. Le carbone organique a été déterminé après oxydation au bichromate de potassium, la capacité d'échange cationique à l'aide de la méthode à la cobaltihexamine [5], et le fer et l'aluminium appartenant au réseau des oxy-hydroxydes après extraction par le réactif de Mehra-Jackson [13]. La teneur en eau aux potentiels de $-0,1$ et $-1,5 \mathrm{MPa}$ ( $W$ en $\mathrm{g}$ d'eau par $\mathrm{g}$ de sol séché à l'étuve à $105 \circ$ C) a été mesurée à l'aide de cellules à pression, les cylindres de cuivre contenant les échantillons ayant été préalablement réhumectés par capillarité et disposés sur une pâte de kaolinite. La géométrie de l'espace poral a été étudiée par porosimétrie au mercure. La tension de surface du mercure et la valeur de l'angle du contact entre le mercure et le solide sont respectivement égaux à $0,484 \mathrm{~N} \cdot \mathrm{m}-1$ et 130 . Enfin, à chaque profondeur échantillonnée, des lames minces ont été confectionnées, et la microstructure a été étudiée en microscopie électronique à balayage en mode électrons rétrodiffusés.

\section{Résultats}

\subsection{Composition des sols étudiés}

L'observation à la loupe binoculaire des fractions limon grossier et sable a montré qu'elles étaient composées, après dispersion, de grains de quartz exempts de 
revêtements argileux et de quelques nodules ferrugineux (2-5\%). Le site de Brasilândia présente des sols sablo-argileux à argilo-sableux, alors qu'ils sont argileux à Planaltina. À Brasilândia, la teneur sols étudiés, alors qu'elle est sensiblement constante dans les sols de Planaltina (tableau). La capacité d'échange cationique est la plus élevée dans I'horizon 0-3 cm et tend ensuite à décroître avec la profondeur, de 3,3 à 1,8 cmolc $\cdot \mathrm{kg}-1$ à Brasilândia et de 9,3 à 2,5 cmolc $\cdot \mathrm{kg}-1$ à Planaltina. La teneur en carbone organique décroît avec la profondeur, mais elle est plus élevée dans les sols de Planaltina. Elle demeure voisine de $15 \mathrm{~g} \cdot \mathrm{kg}-1$ entre 12 et $50 \mathrm{~cm}$ de profondeur à Planaltina, alors qu'elle est voisine de 4 à $8 \mathrm{~g} \cdot \mathrm{kg}-1$ entre 18 et $80 \mathrm{~cm}$ de profondeur à Brasilândia (tableau). Le fer extrait par le réactif de Mehra-Jackson est plus élevé à Planaltina $(56,1-59,1 \mathrm{~g} \cdot \mathrm{kg}-1)$ qu'à Brasilândia $(16,1-30,8 \mathrm{~g} \cdot \mathrm{kg}-1)$. Quant à l'aluminium extrait par cette méthode, il est aussi plus élevé à Planaltina $(10,8-14,6 \mathrm{~g} \cdot \mathrm{kg}-1)$ qu'à Brasilândia $(2,1-3,3 \mathrm{~g} \cdot \mathrm{kg}-1)$.

\subsection{Observations en microscopie électronique}

Les échantillons des sols du site de Brasilândia présentent une phase argileuse, qui est en partie organisée en microagrégats de section sub-circulaire et de 50 à $300 \mu \mathrm{m}$ de diamètre, qui sont juxtaposés aux grains de quartz (figure 1), et pour partie en agrégats de plus grande taille $(2-10 \mathrm{~mm})$, mais aussi de section sub-circulaire. La proportion de microagrégats et leur netteté augmentent avec la profondeur dans les deux sols étudiés (figures 1a et 1c). La compacité de l'assemblage des grains de quartz avec les agrégats décroît avec la profondeur. Cette compacité de l'assemblage est particulièrement élevée sous pâturage entre 6 et $12 \mathrm{~cm}$ de profondeur. Les sols du site de Planaltina paraissent composés essentiellement d'argile qui est organisée sous la forme d'agrégats de différentes tailles (figures $1 d-1 f$ ). Comme pour les sols du site de Brasilândia, on observe la présence de microagrégats, mais ceux-ci appartiennent à deux ensembles : des microagrégats très poreux et d'autres, beaucoup plus compacts. Les premiers sont de plus petite taille $(50-200 \mu \mathrm{m})$ que les seconds $(500-1000 \mu \mathrm{m})$; leurs contours sont moins nets. Les microagrégats compacts sont présents, soit isolés et associés aux agrégats de plus petite taille, soit agglomérés en des volumes compacts de grande taille $(5-20 \mathrm{~mm})$, au sein desquels ils sont reconnaissables par la présence de vides polyconcaves et de vides planaires courbes à leur périphérie (figure 1d). La proportion des microagrégats augmente avec la profondeur dans les deux sols, mais, à chaque profondeur, elle est plus élevée sous cerrado (figure 1e). Sous pâturage, la proportion des volumes compacts composés d'agrégats est particulièrement élevée entre 3 et 40 $\mathrm{cm}$ de profondeur (figure $1 f$ ).

\subsection{Propriétés de rétention en eau et porosité}

Les sols du site de Brasilândia retiennent moins d'eau que ceux du site de Planaltina (tableau). Pour les sols du site de Brasilândia, la quantité d'eau retenue varie de 0,087 à $0,167 \mathrm{~cm} 3 \cdot \mathrm{g}-1$ et de 0,066 à $0,129 \mathrm{~cm} 3 \cdot \mathrm{g}-1$, pour respectivement $-0,1$ et $-1,5 \mathrm{MPa}$. Pour les sols du site de Planaltina, elle varie de 0,248 à $0,304 \mathrm{~cm} 3 \cdot \mathrm{g}-1$ et de 0,223 à $0,246 \mathrm{~cm} 3 \cdot \mathrm{g}-1$ pour respectivement $-0,1$ et $-1,5 \mathrm{MPa}$. Les courbes d'entrée de mercure montrent la présence de deux classes de pores, qui correspondent à des pores de diamètre équivalent $(D e)$ tel que $0,006<D$ e $<0,2 \mu \mathrm{m}$ et $0,2<D e<360 \mu \mathrm{m}$. Seuls les résultats concernant les pores de $D e<3 \mu \mathrm{m}$ seront présentés et discutés dans cette note (tableau). 
Tableau. Caractéristiques physiques et chimiques des sols étudiés : densité apparente (Da) en $\mathrm{g} \cdot \mathrm{cm}-3$; composition granulométrique et teneur en carbone organique (CO) en $\mathrm{g} \cdot \mathrm{kg}-1$; rapport carbone organique sur azote $(\mathrm{C} / \mathrm{N})$; capacité d'échange cationique du sol $(C E C)$ en $\mathrm{cmolc}^{\mathrm{kg}-1}$; teneur en aluminium (Al) et fer $(\mathrm{Fe})$ extrait au citrate-dithionite-bicarbonate en $\mathrm{g} \cdot \mathrm{kg}-1$; volume de pores de diamètre équivalent $<3$ $\mu \mathrm{m}(V \mathrm{p}<3 \mu \mathrm{m})$ et $<0,2 \mu \mathrm{m}(V p<0,2 \mu \mathrm{m})$ en $\mathrm{cm} 3 \cdot \mathrm{g}-1$; teneur en eau à $-0,1 \mathrm{MPa}(W-0,1)$ et $-1,5 \mathrm{MPa}$ $(W-1,5)$ en $\mathrm{cm} 3 \cdot \mathrm{g}-1 ;{ }^{*}$ mesure effectuée pour la profondeur $0-7 \mathrm{~cm}$.

Table. Physicochemical analysis of the soils studied: bulk density $(\mathrm{Da})$ in $\mathrm{g} \cdot \mathrm{cm}-3$; particle-size-distribution and organic carbon $(\mathrm{CO})$ in $\mathrm{g} \cdot \mathrm{kg}-1$; organic-carbon nitrogen ratio $(\mathrm{C} / \mathrm{N})$; cation exchange capacity $(C E C)$ in cmolc $\cdot \mathrm{kg}-1$; aluminium (Al) and iron $(\mathrm{Fe})$ extractable with citrate-dithionite-bicarbonate reactant in $\mathrm{g} \cdot \mathrm{kg}-1$; volume of pores with equivalent pore diameter $<3 \mu \mathrm{m}(V \mathrm{p}<3 \mu \mathrm{m})$ and $<0.2 \mu \mathrm{m}$ in $\mathrm{cm} 3 \cdot \mathrm{g} 1(V p<$ $0.2 \mu \mathrm{m})$; water content at $-0.1 \mathrm{MPa}(W-0.1)$ and $-1.5 \mathrm{MPa}(W-1.5)$ in $\mathrm{cm} 3 \cdot \mathrm{g}-1)$; * ${ }^{*}$ easurement between 0 and $7 \mathrm{~cm}$ depth.

\begin{tabular}{|c|c|c|c|c|c|c|c|c|c|c|c|c|c|c|}
\hline \multirow{2}{*}{$\begin{array}{l}\text { Profondeur } \\
\text { (cm) }\end{array}$} & \multirow[t]{2}{*}{$D_{2}$} & \multicolumn{3}{|c|}{ Granulomémie ( $\mu \mathrm{m})$} & \multirow[t]{2}{*}{$\mathrm{CO}$} & \multirow[t]{2}{*}{$\mathrm{CN}$} & \multirow[t]{2}{*}{$\mathrm{pH}$} & \multirow[t]{2}{*}{$C E C$} & \multirow[t]{2}{*}{ Al } & \multirow[t]{2}{*}{$\mathrm{Fe}$} & \multirow[t]{2}{*}{$V_{\mathrm{p}}<3 \mathrm{\mu m}$} & \multirow[t]{2}{*}{$V_{\mathrm{P}}<0,2 \mu \mathrm{m}$} & \multirow[t]{2}{*}{$W_{-0,1}$} & \multirow[t]{2}{*}{$W_{-1,5}$} \\
\hline & & $<2$ & $2-50$ & $50-2000$ & & & & & & & & & & \\
\hline \multicolumn{15}{|c|}{ Brasilândia-Cerrado } \\
\hline $0-3$ & 0,80 & 321 & 86 & 593 & 20,1 & 14,0 & 3,9 & 3,1 & 3,2 & 27,7 & 0,108 & 0,083 & 0,158 & 0,121 \\
\hline $3-6$ & 1,03 & 353 & 72 & 576 & 14,2 & 12,7 & 3,9 & 2,6 & 3,3 & 28,0 & 0,120 & 0,102 & 0,167 & 0,129 \\
\hline $6-15$ & 1,03 & 305 & 84 & 611 & 10,9 & 12,2 & 3,9 & 2,0 & 3,2 & 26,6 & 0,098 & 0,082 & 0,143 & 0,116 \\
\hline $18-30$ & 1,02 & 303 & 111 & 586 & 7,5 & 12,4 & 4,0 & 2,4 & 2,9 & 27,8 & 0,100 & 0,087 & 0,131 & 0,109 \\
\hline $30-40$ & 1,03 & 310 & 47 & 643 & - & - & - & - & - & - & 0,100 & 0,089 & - & - \\
\hline $60-80$ & 0,94 & 365 & 143 & 491 & 4,1 & 10,9 & 4,2 & 2,5 & 2,8 & 30,8 & 0,119 & 0,105 & 0,132 & 0,117 \\
\hline $90-100$ & 0,92 & 432 & 54 & 515 & - & - & - & - & - & - & 0,129 & 0,111 & - & - \\
\hline \multicolumn{15}{|c|}{ Brasilândia-Pâturage } \\
\hline $0-3$ & 1,08 & 186 & 40 & 775 & 14,2 & 14,7 & 5,8 & 3,3 & 2,1 & 16,1 & 0,063 & 0,046 & 0,087 & 0,066 \\
\hline $3-6$ & 1,17 & 212 & 45 & 742 & 11,7 & 13,6 & 5,3 & 2,6 & 2,5 & 19,0 & 0,070 & 0,055 & 0,089 & 0,069 \\
\hline $10-13$ & 1,22 & 215 & 66 & 719 & 9,2 & 14,3 & 5,2 & 2,2 & 2,6 & 19,9 & 0,072 & 0,058 & 0,091 & 0,072 \\
\hline $14-17$ & - & 221 & 64 & 715 & 7,9 & 13,3 & 5,3 & 2,2 & 2,3 & 20,4 & 0,076 & 0,065 & 0,092 & 0,075 \\
\hline $18-30$ & 1,21 & 230 & 92 & 678 & 6,2 & 13,7 & 5,2 & 2,0 & 2,8 & 25,6 & 0,079 & 0,068 & 0,096 & 0,079 \\
\hline $30-40$ & 1,09 & 264 & 47 & 689 & - & - & - & - & - & - & 0,085 & 0,074 & - & - \\
\hline $60-80$ & 1,11 & 306 & 60 & 635 & 3,6 & 13,0 & 5,0 & 1,8 & 2,5 & 22,3 & 0,104 & 0,084 & 0,108 & 0,093 \\
\hline $90-100$ & 1,02 & 328 & 44 & 698 & - & - & - & - & - & - & 0,100 & 0,088 & - & - \\
\hline \multicolumn{15}{|c|}{ Planaltina-Cerrado } \\
\hline $0-3$ & - & 775 & 123 & 102 & 29,2 & 15,5 & 5,0 & 3,7 & 14,4 & 56,4 & 0,238 & 0,199 & 0,304 & 0,242 \\
\hline $3-6$ & $1,07^{*}$ & 764 & 132 & 105 & 24,1 & 14,3 & 5,1 & 2,5 & 14,6 & 57,7 & 0,240 & 0,208 & 0,289 & 0,240 \\
\hline $12-15$ & 1,09 & 792 & 124 & 77 & 18,9 & 15,0 & 5,1 & 2,9 & 14,1 & 56,1 & 0,233 & 0,206 & 0,275 & 0,246 \\
\hline $27-30$ & 1,13 & 773 & 142 & 85 & 14,0 & 14,2 & 5,3 & 2,8 & 14,3 & 57,2 & 0,234 & 0,214 & 0,271 & 0,237 \\
\hline $35-38$ & 1,06 & 775 & 136 & 89 & 12,5 & 15,3 & 5,2 & 2,8 & 13,9 & 57,4 & 0,239 & 0,216 & 0,262 & 0,237 \\
\hline $90-100$ & 1,00 & 782 & 139 & 78 & 6,7 & 14,9 & 5,4 & 2,5 & 13,3 & 57,7 & 0,246 & 0,222 & 0,260 & 0,238 \\
\hline \multicolumn{15}{|c|}{ Planaltina-Pâturage } \\
\hline $0-3$ & - & 679 & 248 & 73 & 28,0 & 17,6 & 7,0 & 9,3 & 10,8 & 56,7 & 0,225 & 0,189 & 0,292 & 0,237 \\
\hline $3-6$ & $1,17^{*}$ & 722 & 211 & 68 & 20,2 & 15,1 & 5,7 & 4,4 & 11,9 & 59,1 & 0,229 & 0,191 & 0,295 & 0,229 \\
\hline $12-15$ & 1,14 & 737 & 201 & 63 & 16,3 & 14,1 & 5,2 & 3,2 & 11,7 & 59,0 & 0,217 & 0,194 & 0,258 & 0,229 \\
\hline $25-32$ & 1,21 & 669 & 261 & 70 & 15,9 & 13,6 & 5,2 & 3,2 & 11,8 & 57,9 & 0,212 & 0,196 & 0,258 & 0,223 \\
\hline $33-40$ & 1,21 & 698 & 233 & 69 & 15,2 & 13,7 & 5,2 & 3,4 & 12,1 & 58,5 & 0,216 & 0,197 & 0,248 & 0,226 \\
\hline $48-51$ & 1,25 & 696 & 234 & 70 & 14,4 & 13,4 & 5,3 & 3,0 & 11,6 & 57,1 & 0,218 & 0,197 & 0,249 & 0,223 \\
\hline $90-100$ & 1,13 & 728 & 206 & 67 & 6,3 & 12,8 & 5,2 & 2,5 & 11,2 & 58,3 & 0,226 & 0,202 & 0,268 & 0,229 \\
\hline
\end{tabular}

\section{Discussion}

\subsection{Microagrégation et activité biologique}

Les microagrégats de section subcirculaire et de 200 à $500 \mu \mathrm{m}$ de diamètre qui ont été décrits dans les sols étudiés correspondent aux microagrégats décrits pour l'horizon ferralique [11] ; ils résulteraient de l'activité des termites [7, 15]. Les microagrégats de 
plus grande taille, observés isolés ou agglomérés au sein de volumes compacts, sont des déjections de vers géophages de petite taille en cours de détermination. Les assemblages compacts d'agrégats sont très abondants entre 3 et $40 \mathrm{~cm}$ de profondeur sous pâturage dans le site de Planaltina. Dans ce site, le changement de végétation s'est traduit par une perte de diversité taxonomique de la faune d'invertébrés du sol [1]. Celle-ci aurait provoqué une transformation physique du sol, à la suite de la forte augmentation de la densité d'une espèce de vers, comme cela a été observé en Amazonie [4]. Dans le sol sous pâturage du site de Brasilândia, les agrégats de 2 à 10 $\mathrm{mm}$ de diamètre observés en microscopie seraient l'équivalent des volumes compacts composés de microagrégats dans les sols du site de Planaltina. Leur présence en plus grand nombre dans le sol sous pâturage serait aussi à interpréter comme étant une conséquence du défrichement et de la mise en culture.
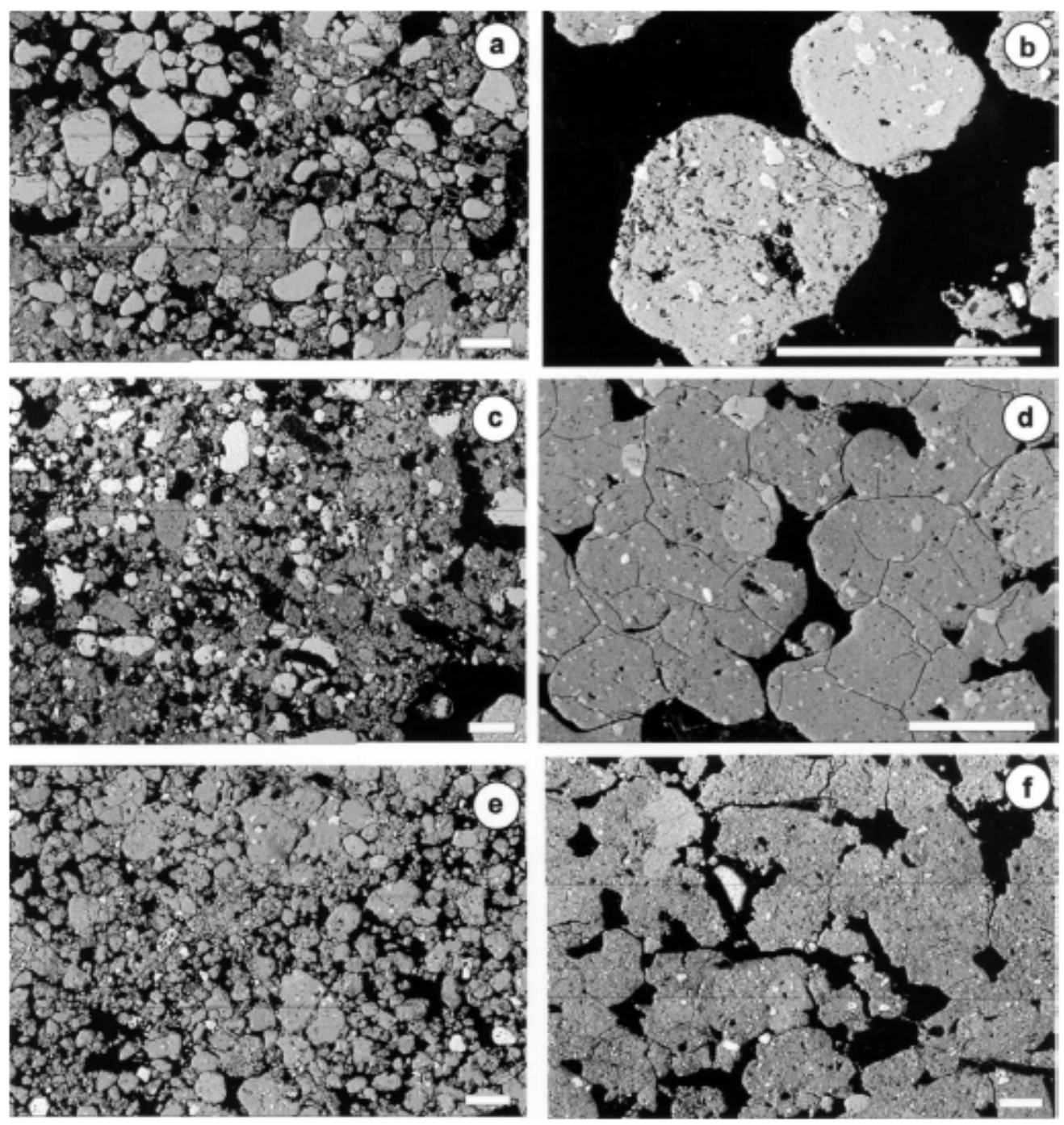

Figure 1. Mode d'assemblage des microagrégats observés en microscopie électronique à balayage (mode électrons rétrodiffusés), site de Brasilândia (horizon 3-6 $\mathrm{cm}$ sous cerrado, $\mathbf{a}$ et $\mathbf{b}$, horizon 75-80 $\mathrm{cm}$ sous pâturage, c) et site de Planaltina (horizons $3-6 \mathrm{~cm}$, d, et 75-80 cm, e, sous cerrado, horizon 3-6 $\mathrm{cm}$ sous pâturage, f). Les vides occupés par de la résine sont en noir ; les plages gris clair correspondent 
aux grains de quartz ; les plages hétérogènes en gris foncé correspondent à la phase argileuse et à sa porosité associée (échelle $=500 \mu \mathrm{m}$ ). Figure 1. Microaggregates packing in scanning electron microscopy (back-scattered electrons), site of Brasilândia (horizon 3-6 cm under cerrado, a and b, horizon 75-80 cm under pasture, c) and site of Planaltina (horizons 3-6 cm, d, and 75-80 cm, e, under cerrado, and horizon 3-6 $\mathrm{cm}$ under pasture, $\mathbf{f}$ ). The black areas are voids occupied by resin, the light grey areas are quartz grains and the heterogeneous dark grey areas correspond to clay phase and associated porosity (bar scale $=500 \mu \mathrm{m})$.

\subsection{Stabilité de l'assemblage lors de la dessiccation}

On observe une relation étroite et positive entre la quantité d'eau retenue aux potentiels de $-0,1$ et $-1,5 \mathrm{MPa}$ et la teneur en argile (respectivement $r 2=0,95$ et 0,99 ). Ainsi, l'eau retenue à ces valeurs de potentiel peut être considérée comme localisée dans des pores résultant de l'assemblage des particules d'argile. Ces pores sont ceux contenus dans les microagrégats. À $-0,1$ et $-1,5 \mathrm{MPa}$, on peut d'ailleurs considérer que l'eau est retenue par des pores de diamètres équivalents respectivement inférieurs à 3 et $0,2 \mu \mathrm{m}$ [14]. Comme cela a été fait antérieurement [2,8], nous pouvons comparer le volume d'eau retenu à $-0,1$ et $-1,5 \mathrm{MPa}$ aux volumes de pores de De respectivement inférieurs à 3 et $0,2 \mu \mathrm{m}$, déterminés par porosimétrie au mercure. Pour les sols étudiés, la teneur en eau à $-0,1 \mathrm{MPa}$ est toujours supérieure au volume de pores de $D e \leq 3 \mu \mathrm{m}$. De même, la teneur en eau à $-1,5 \mathrm{MPa}$ est supérieure au volume de pores de $D e \leq 0,2 \mu \mathrm{m}$. Ainsi, le volume poral est à l'état sec de 3,7 à $31,7 \%$ inférieur à sa valeur à $-0,1 \mathrm{MPa}$ et de 6,6 à $29,9 \%$ inférieur à sa valeur à $-1,5 \mathrm{MPa}$. On en conclut que le volume de pores de $D e \leq 3 \mu \mathrm{m}$ décroît entre $-0,1 \mathrm{MPa}$ et l'état sec ; cette décroissance entraînerait une réorganisation du mode d'assemblage des particules d'argile et, à une échelle moins microscopique, une diminution du volume des microagrégats et l'apparition de fissures, lorsque ceux-ci sont en assemblage compact (figure 1d). Bien que la proportion de microagrégats des deux types varie au sein des profils de sol étudiés (figure 1), aucune différence n'a été relevée en fonction de la proportion de microagrégats des deux types et de leur mode d'assemblage. C'est le cas, en particulier, des échantillons provenant des 40 premiers centimètres des sols sous cerrado et sous pâturage, les sols sous cerrado ayant une proportion élevée de microagrégats à cette profondeur, alors que, sous pâturage, la microstructure est plus continue et la proportion de microagrégats isolés plus faible.

\subsection{Déformation du mode d'assemblage des particules d'argile et teneur en carbone organique}

Lorsque l'on analyse la variation du volume de pores de la phase argileuse entre les potentiels de $-0,1$ et $-1,5 \mathrm{MPa}$ et l'état $\mathrm{sec}$, on observe qu'elle n'est liée, ni à la profondeur, ni à la teneur en argile, mais, en revanche, à la teneur en carbone organique (figure 2). La variation du volume de pores croît en effet avec la teneur en carbone organique des échantillons. Compte tenu des valeurs du rapport $\mathrm{C} / \mathrm{N}$ (tableau), on peut en déduire que le carbone organique appartient majoritairement à une matière organique suffisamment évoluée pour que l'on puisse faire l'hypothèse qu'elle est étroitement associée à la phase argileuse. Des observations en microscopie électronique à transmission sur des Ferralsols d'Amazonie ont en effet montré qu'une partie de l'espace interparticulaire était occupée par de la matière organique [8]. 
La matière organique interviendrait directement dans les mécanismes à l'origine de la variation de volume. Il pourrait s'agir de molécules hydrophiles, qui se réorganiseraient entre $-0,1 \mathrm{MPa}$ et l'état sec et dont la réorganisation entraînerait une importante diminution du volume de pores au sein de la phase argileuse, à la suite d'une évolution vers un assemblage plus compact des particules d'argile.
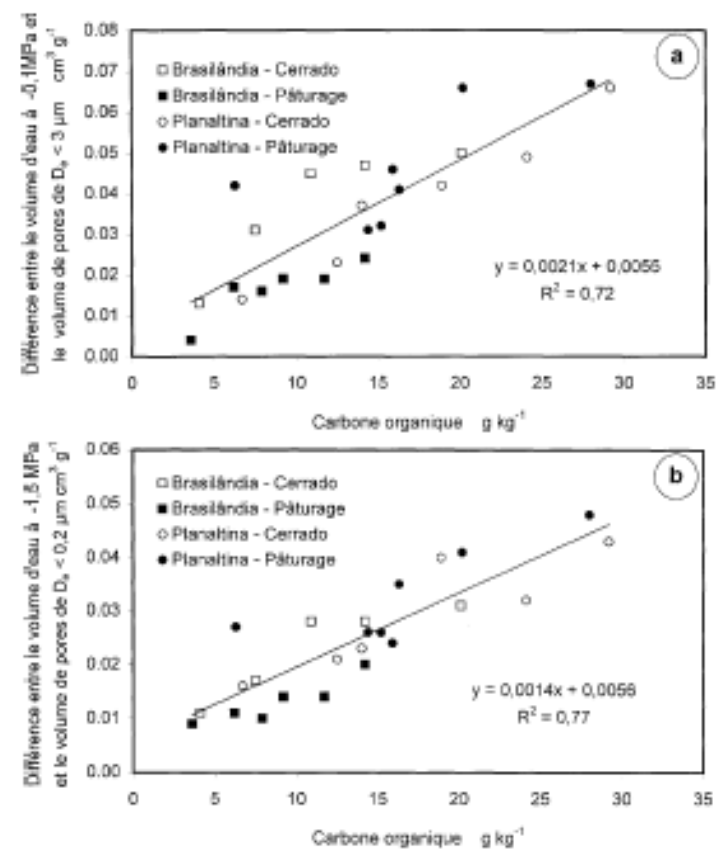

Figure 2. Différence entre le volume d'eau retenu à $-0,1 \mathrm{MPa}$ et le volume de pores de $D e \leq 3 \mu \mathrm{m}$ à l'état sec (a) et différence entre le volume d'eau retenu à $-1,5 \mathrm{MPa}$ et le volume de pores de $D \mathrm{e} \leq 0,2 \mu \mathrm{m}$ à l'état sec (b) en fonction de la teneur en carbone organique des échantillons.

Figure 2. Difference between the volume of water retained at $-0.1 \mathrm{MPa}$ and the volume of pores with $\mathrm{De}$ $\leq 3 \mu \mathrm{m}$ in the dried state (a) and difference between the volume of water retained at $-1.5 \mathrm{MPa}$ and the volume of pores with $D \mathrm{e} \leq 0.2 \mu \mathrm{m}$ in the dried state (b) versus the organic carbon content of the samples.

\section{Conclusion}

Les résultats présentés dans cette note montrent que le mode d'assemblage des particules d'argile au sein des microagrégats de Ferralsols étudiés varie au cours de la dessiccation. Or, la stabilité élevée de ces microagrégats (faible dispersion au contact de l'eau) qui a été mise en évidence dans de nombreuses études permettait de penser qu'il s'agissait d'édifices rigides. Par conséquent, nos résultats indiquent que la stabilité de l'assemblage ne s'oppose pas à sa déformation au cours de la dessiccation. Nos résultats montrent aussi que la déformation de l'assemblage varie indépendamment de la morphologie des microagrégats, de leur mode d'assemblage, de la profondeur et de la teneur en argile. Cette déformation de la phase argileuse lors de la dessiccation ainsi que la diminution de volume poral qui en résulte sont, par conséquent, une propriété physique qui lui est spécifique et, de la sorte, indépendante de son organisation en microagrégats. 
La déformation de l'assemblage est, en revanche, étroitement liée à la teneur en carbone organique et elle est d'autant plus élevée que la teneur en carbone organique est élevée. Un tel résultat indique que la déformation serait directement sous la dépendance de la réorganisation de molécules organiques lors de la dessiccation, lesquelles molécules seraient intimement associées aux particules d'argile.

Remerciements. Cette étude a été réalisée dans le cadre du projet Deresam (Dégradation et réhabilitation de savanes cultivées d'Amérique du Sud) de l'Empresa Brasileira de Pesquisa Agropecuária (Embrapa) et de l'Institut de recherche pour le développement (IRD).

\section{Références}

[1] Benito N.P., Bobillier B., Brossard M., Pasani A., Impacto do desmatamento e da renovação de pastagem sobre a macrofauna edáfica do solo no Cerrado, International Symposium 'Soil functioning under pastures in intertropical areas', Brasília, 16-20 October, CDRom Embrapa Cerrados/IRD, 2000.

[2] Bruand A., Prost R., Effect of water content on the fabric of a soil material: an experimental approach, J. Soil Sci. 38 (1987) 461-472.

[3] Camargo O.A., Moniz A.C., Jorge J.A., Valadares J.M.A.S., Métodos de análise química, mineralógica e física de solos do Instituto Agronômico de Campinas, Instituto Agronômico, Campinas, Boletim Técnico, Campinas, SP, 1986.

[4] Chauvel A., Grimaldi M., Barros E., Blanchard E., Desjardins T., Sarrazin M., Lavelle P., Pasture damage by an Amazonian earthworm, Nature 398 (1999) 32-33.

[5] Ciesielski H., Sterckeman T., Determination of cation exchange capacity and exchangeable cations in soils by means of cobalt hexamine trichloride. Effects of experimental conditions, Agronomie 17 (1997) 1-7.

[6] EMBRAPA, Centro Nacional de Pesquisa de solos (Rio de Janeiro, RJ), Sistema Brasileiro de Classificação de Solos, Embrapa Produção de Informação, Brasilia, Embrapa Solos, Rio de Janeiro, 1999.

[7] Eschenbrenner V., Contribution des termites à la microagrégation des sols tropicaux, Cah. Orstom, sér. Pédol. 22 (1986) 397-408.

[8] Grimaldi M., Sarrazin M., Chauvel A., Luizao F., Nunes N., Rodriguez M.R.L., Amblard P., Tessier D., Effets de la déforestation et des cultures sur la structure des sols argileux d'Amazonie brésilienne, Cahiers Agricultures 2 (1993) 36-47.

[9] Hartmann C., Évolution et comportement de sols sabloargileux ferrallitiques sous culture de palmiers à huile. Cas de la plantation R. Michaux à Abou (Côte d'Ivoire), thèse, université Paris 6, 1991.

[10] ISSS Working Group R.B., in : Deckers J.A., Nachtergaele F.O., Spaargaren O.C. (Eds.), World Reference Base for Soil Resources: Introduction, 1st ed., International Society of Soil Science (ISSS), International Soil Reference and Information Centre (ISRIC) and Food and Agriculture Organization of the United Nations (FAO), Acco, Leuven, 1998.

[11] Klamt E., Van Reeuwijk L.P., Evaluation of morphological, physical and chemical characteristics of Ferralsols and related soils, R. Bras. Ci. Solo 24 (2000) 573-587. 
[12] Koutika L.-S., Bartoli F., Andreux F., Cerri C.C., Burtin G., Choné T., Philippy R., Organic matter dynamics and aggregation in soils under rain forest and pastures of increasing age in the eastern Amazon Basin, Geoderma 76 (1997) 87-112.

[13] Mehra O.P., Jackson M.L., Iron oxide removal from soil and clays by a dithionitecitrate system buffered with sodium bicarbonate, Clays Clay Miner. 7 (1960) 317-327.

[14] Murray R.S., Quirk J., Clay-water interactions and the mechanism of soil swelling, Colloids Surfaces 1 (1980) 17-32.

[15] Oliveira C.V., Ker J.C., Duarte M.N., Curi N., Fontes L.E.F., Atributos micromorfológicos de solos do projeto Jaíba, Norte de Minas Gerais, R. Bras. Ci. Solo 24 (2000) 117-128.

[16] Pédro G., Chauvel A., Melfi A., Recherches sur la constitution des Terra Roxa Estructurada du Brésil, Ann. Agron. 27 (1976) 265-294.

[17] Pinheiro-Dick D., Schwertmann U., Microaggregates from Oxisols and Inceptisols: dispersion through selective dissolutions and physicochemical treatments, Geoderma 74 (1996) 49-63. 